\title{
Gamma rays from supernova remnants: time evolution
}

\section{Daniele Gaggero*}

GRAPPA Institute, University of Amsterdam, 1098 XH Amsterdam, The Netherlands

E-mail: d.gaggero@uva.nl

\section{Stefano Gabici}

APC, Univ. Paris Diderot, CNRS/IN2P3, CEA/Irfu, Obs. de Paris, Sorbonne Paris Cité, 75013

Paris, France

E-mail: gabicieapc.in2p3.fr

\section{Fabio Zandanel}

GRAPPA Institute, University of Amsterdam, 1098 XH Amsterdam, The Netherlands

E-mail: f.zandaneleuva.nl

Galactic cosmic rays are believed to be accelerated at supernova remnant shocks. We model the dynamical evolution of type Ia and type II supernova remnants, and discuss how the maximum energy of accelerated protons and electrons is expected to evolve in time. In particular, we discuss whether supernova remnants can accelerate protons all the way up to $\mathrm{PeV}$ energies, and the crucial role of of magnetic field amplification in this context. The efficiency of field amplification is modeled in a phenomenological way, but some theoretical frameworks that may explain this mechanism are examined (in particular, the non-resonant, current-driven instability proposed by Bell), with emphasis on the role of the slope of the CR spectrum. In the final part we present the predicted evolution of the SNR gamma-ray spectrum for two benchmark cases of SNR I and II, we discuss in detail the impact of several crucial parameters (gas density, magnetic field inside the remnant, structure of the interstellar medium surrounding the SNR), and eventually compare our predictions with current observations of young SNRs in our Galaxy. 


\section{Introduction}

Understanding the nature of the Galactic sources able to accelerate cosmic rays (CRs) up to $\mathscr{O}(P V)$ rigidities is one of the main goals in the Astroparticle research field. The crucial role of supernova remnants (SNRs) was first outlined in [Baade \& Zwicky 1934] out of energy budget arguments; two decades later the picture was better defined in term of SNRs located in our Galaxy [Ter Haar 1950, Morrison 1957] - although a mechanism able to provide an effective CR acceleration at SNR shocks had not been proposed yet at that time. A detailed theory of SNR diffusive shock acceleration was developed by Bobalsky ${ }^{1}$. It was soon realized that a significant amplification of the magnetic field at the shock is required to reach PeV energies. To test the theory and identify whether SNRs are actually able to accelerate hadrons up to PV rigidities, and how long this process can be sustained, it is useful to investigate the broad-band non-thermal radiation emitted by these objects, from radio waves all the way up to gamma rays. On the observational side, we are gathering interesting data at all wavelengths (especially for middle-aged SNRs). In particular, the current generation of Cherenkov gamma-ray telescopes have detected a population of $16 \mathrm{TeV}$ sources associated with shell-type SNRs. However, these objects are quite old and none of these - including the bright, well-studied RX J1713.7 - 3946 - show striking evidence of ongoing PeV acceleration. The only claim of a gamma-ray emission with no clear cutoff in the multi-TeV domain comes from the Galactic center region [HESS Collaboration et al. 2016] (see [Gaggero et al. 2017, Jouvin et al. 2017] for different interpretations).

On the other hand, on the modeling side, despite this large collection of data, a clear comprehension of the crucial aspects of CR acceleration outlined above is still lacking. In this contribution we present a systematic study on the evolution of the gamma-ray emission from SNRs as a function of time, with the future prospect of a detailed comparison with an even more extended and accurate set of $\mathrm{GeV}$ to multi-TeV data regarding a large population of young and middle-aged SNR.

\section{Dynamical evolution of supernova remnants}

The dynamical evolution of a SNR shell can be divided into three temporal phases: the ejectadominated, the Sedov (which starts as soon as the mass of the interstellar gas swept up by the shock equals the mass of the stellar matter ejected in the supernova explosion), and the radiative phase. Here, we focus on the two earlier ones, where most of the CR acceleration is expected to happen.

For both type Ia and type II supernovae, we compute the time evolution of the shock radius and velocity in the ejecta-dominated phase by evaluating the self-similar expressions presented in [Chevalier 1982]. Those scaling relations only depend on $E_{51}$ (the supernova explosion energy in units of $10^{51} \mathrm{erg}$ ), $M_{e j, \odot}$ (the mass of the ejecta in solar masses), and $n$ (the density of the interstellar medium in $\mathrm{cm}^{-3}$, in the SN I case). In the SN II case the self-similar expressions also depend on $\dot{M}$ and $u_{w}$, i.e. the mass-loss rate and the wind velocity, respectively.

In order to model the evolution of the shock radius and velocity in the Sedov phase we adopt instead the widely used thin-shell approximation [Ostriker \& McKee 1988], based on the assumption that the mass is mostly concentrated in a thin spherical shell at the forward shock, i.e. at $r=R_{s}$.

\footnotetext{
${ }^{1}$ Thierry Montmerle's abbreviation for Blandford, Ostriker [Blandford \& Ostriker 1978], Bell [Bell 1978], Axford, Leer, Skadron [Axford et al. 1977] and Krymsky [Krymskii 1977].
} 
For non-radiative systems, the total energy is conserved and is equal to the energy released in the explosion $E_{\mathrm{SN}}$. Given energy conservation, that quantity is the sum of the kinetic energy of the shell and the total thermal energy in the remnant:

$$
E_{\mathrm{SN}}=\frac{1}{2} M u_{s}^{2}+\frac{4 \pi}{3} R_{s}^{3} \frac{P_{\text {in }}}{\gamma+1}
$$

where $P_{i n}$ is the gas pressure in the SNR, $\gamma$ the gas adiabatic index, and $M$ the mass of the shell:

$$
M=M_{e j}+4 \pi \int_{0}^{R_{s}} \rho(r) r^{2} \mathrm{~d} r
$$

where the first term on the right-hand side represents the mass of the ejecta and the second the mass of the interstellar medium (of mass density $\rho$ ) swept up by the shock. The equation of momentum conservation is:

$$
\frac{\mathrm{d}}{\mathrm{d} t}\left(M u_{s}\right)=4 \pi R_{s}^{2} P_{\text {in }}
$$

The three equations above define the dynamics of the SNR until the shock becomes radiative, and cooling cannot be neglected anymore, or subsonic. In order to solve Eq.s 2.1, 2.2, and 2.3 and get the time evolution of the SNR shock position and velocity, we need to specify the profile of the ambient density around the supernova, $\rho(r)$. For a type Ia supernova we assume a homogeneous ambient medium of density $\rho=\mu n m_{p}$ where $m_{p}$ is the proton mass and $\mu m_{p} \sim 1.4 m_{p}$ the mean interstellar atom mass per $\mathrm{H}$ nucleus. In a type II supernova, instead, the SNR shock propagates through the red supergiant wind $\rho \propto r^{-2}$, then through a tenuous bubble inflated by the wind of the massive progenitor star in main sequence, and finally in the interstellar medium [Ptuskin \& Zirakashvili 2005, Cristofari et al. 2013]. The typical radius of the wind $R_{\mathrm{w}}$ is estimated by equating the ram pressure of the wind itself $P_{\text {ram }}=\dot{M} u_{\mathrm{w}} /\left(4 \pi r^{2}\right)$ to the thermal pressure of the bubble interior [Parizot et al. 2004] and is of the order of few parsecs. The radius of the tenuous hot bubble is $R_{\mathrm{b}}=28\left(L_{36} / n_{0}\right)^{1 / 5} t_{\mathrm{Myr}}^{3 / 5} \mathrm{pc}$, where $L_{36}$ is the main-sequence star wind power in units of $10^{36} \mathrm{erg} / \mathrm{s}, n_{0}$ the density of the interstellar medium outside of the bubble, and $t_{\mathrm{Myr}}$ is the wind lifetime in units of mega-years. The density inside the bubble is $n_{\mathrm{b}}=0.01\left(L_{36}^{6} n_{0}^{19} t_{\mathrm{Myr}}^{-22}\right)^{1 / 35} \mathrm{~cm}^{-3}$ and the gas temperature $T_{b}=1.6 \times 10^{6}\left(L_{36}^{8} n_{0}^{2} t_{\mathrm{Myr}}^{-6}\right)^{1 / 35} \mathrm{~K}$ [Castor et al. 1975].

\section{Maximum energy of accelerated particles}

It is well known that the acceleration of CRs up to PeV energies at SNR shocks requires a significant amplification of the magnetic field [Hillas 2005]. Indeed, this picture is supported by X-ray observations of several young SNRs, which revealed magnetic fields whose strengths are well in excess of the typical interstellar value [Vink 2012].

Bell [Bell 2004] proposed that the upstream magnetic field can be highly amplified by a non-resonant instability driven by the CR flux escaping the shock. Another possibility is to amplify the upstream field as the result of the turbulence induced by the upstream CR gradient acting on an inhomogeneous ambient medium. This mechanism is known as Drury instability [Drury \& Downes 2012]. A third mechanism was proposed by Giacalone and Jokipii [Giacalone 2007]: Here the idea is that pre-existing turbulent density fluctuations upstream of the shock can provide the field amplification. 
The effectiveness of such mechanisms in amplifying the magnetic field to the level needed to accelerate $\mathrm{CRs}$ up to the $\mathrm{PeV}$ domain is still debated. In particular, according to the discussion in [Gabici et al. 2016], and in agreement with the findings of Schure and Bell [Schure \& Bell 2013], it can be shown that, in the framework of Drury field amplification, CR acceleration up to $\mathrm{PeV}$ energies is possible only in the very early phase of the SNR evolution, and gets less and less efficient for steeper spectra. A similar result holds in the other proposed scenarios. However, observations of young SNRs suggest that few percent of the shock pressure is indeed converted into magnetic field pressure downstream of the shock [Völk et al. 2005]: Therefore, in the following, we assume that field amplification is active and efficient, and that $3.5 \%$ of the ram pressure is actually converted into magnetic pressure. If this is the case, then CRs can be accelerated up to the energy of the CR knee at the transition between the ejecta-dominated and Sedov phase of the SNR evolution [Zirakashvili \& Ptuskin 2012]. Based on this evidence, in the following we develop a recipe to estimate the maximum energy of CR accelerated at a SNR shock of a given age.

The maximum energy of particles accelerated at the shock of a SNR of age $t_{\text {age }}$ is then given by the most stringent among the following conditions:

- age-limited acceleration: The acceleration rate is given by [Drury 1983]:

$$
\frac{\mathrm{d} p}{\mathrm{~d} t}=\frac{\Delta p}{\Delta t} \sim 0.11 \frac{u_{s}^{2}}{D_{1}} p
$$

where $p$ is the particle momentum, $D_{1}$ is the upstream particle diffusion coefficient, $u_{s}$ is the shock velocity. Equation 3.1 can be integrated up to a time $t_{\text {age }}$ and give the maximum energy of accelerated particles in the absence of particle energy losses and escape from the system [Lagage \& Cesarsky 1983];

- escape-limited acceleration: the maximum energy due to particle escape is estimated by equation the diffusion length of particles ahead of the shock $l_{d}=u_{s} / D$, to some fraction, typically $\chi \approx 0.05 \ldots 0.1$ of the SNR shock radius [Ptuskin \& Zirakashvili 2005]. In the following we will adopt the value $\chi=0.05$;

- loss-limited acceleration: while the acceleration of protons at SNR shocks proceeds unimpeded by energy losses, the acceleration of electrons can be limited by synchrotron radiative losses acting at a rate $\mathrm{d} p /\left.\mathrm{d} t\right|_{s}$. The maximum energy of accelerated electrons can then be derived by equating the momentum gain per cycle $\left(\Delta p=\frac{4}{3} \frac{u_{1}-u_{2}}{v} p\right)$ to the momentum loss per cycle, given by $\left.\Delta p\right|_{s}=\mathrm{d} p /\left.\mathrm{d} t\right|_{s, 1} \Delta t_{1}+\mathrm{d} p /\left.\mathrm{d} t\right|_{s, 2} \Delta t_{2}$, where the subscripts refer to the upstream (1) and downstream (2) region of the shock and $\Delta t_{i}=4 D_{i} / v u_{i}$ with $i=1,2$ [Vannoni et al. 2009].

The upper panels of Fig. 1 show the value of the magnetic field upstream of the SNR shock as a function of the SNR age.

\section{Evolution of the CR spectrum: Our setup}

Given the scenario discussed above, we model the evolution of the leptonic and hadronic CR spectrum inside the remnant. We follow the evolution of the remnant for $\sim 10^{5}$ years, with timestep 
Table 1: Parameters adopted to describe the evolution of supernovae of type Ia and II. Columns 2 to 8 refer to the supernova explosion energy, the mass of the ejecta, the density of the interstellar medium, the red supergiant wind mass loss rate and velocity, and the main sequence wind power and duration.

\begin{tabular}{cccccccc}
\hline type & $E_{51}$ & $M_{e j, \odot}$ & $n$ & $\dot{M}_{-5}$ & $u_{w, 6}$ & $L_{36}$ & $t_{\mathrm{Myr}}$ \\
\hline Ia & 1 & 1.4 & 0.1 & - & - & - & - \\
II & 1 & 3 & - & 1 & 1 & 1 & 4 \\
\hline
\end{tabular}
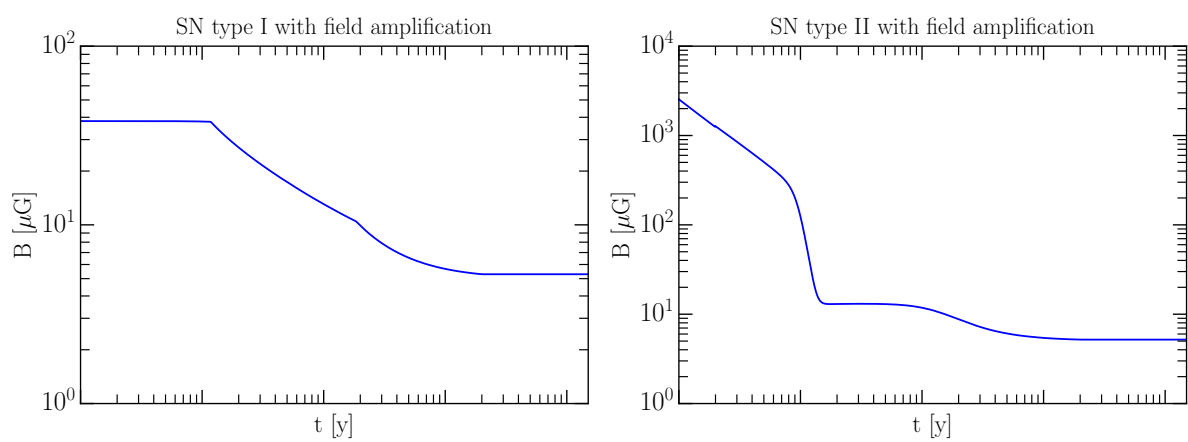

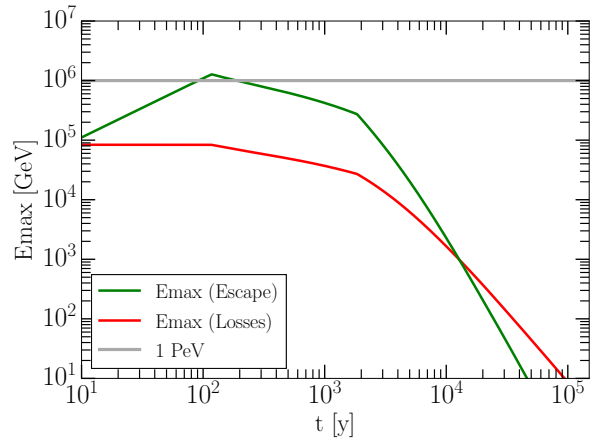

(a) $\mathrm{SN}$ Ia

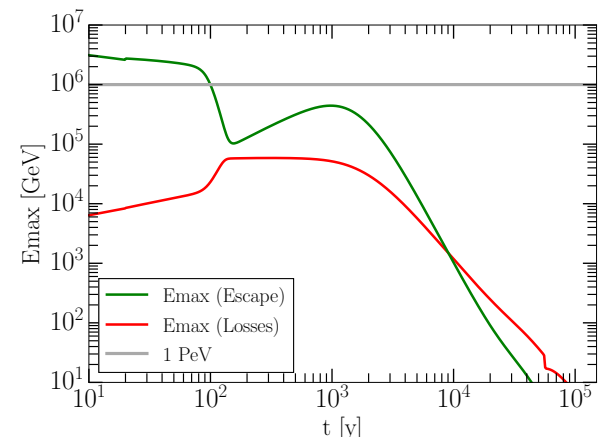

(b) SN II

Figure 1: Upper row: magnetic field strength upstream of the shock of a type Ia and II supernova, as a function of the SNR age. Lower row: maximum energy of accelerated particles at the shock. For CR protons the maximum energy is determined by particle escape from the shock (green lines), while for electrons the maximum energy is determined either by escape or by synchrotron losses (red lines).

$\simeq 1$ year. At each timestep, we inject electrons and protons according to an unbroken power-law $Q=Q_{0} E^{-\alpha} e^{-\left(E / E_{\text {cut }}\right)}$ where we fix $\alpha=2.3$, and we set the exponential cutoff $E_{\text {cut }}$ at the maximal energy, computed as described above. In particular, we recall that, for protons, the escape-limited maximal energy is dominant; for the electrons, instead, the minimum value between the lossdominated and escape-dominated $E_{\max }$ has to be taken into account. The normalization term $Q_{0}$ is set at $0.1 \%$ of the incoming energy flux $f$, defined as $f=1 / 2 \rho u_{s}^{3} \cdot 4 \pi R_{s}^{2}$. We solve numerically the time evolution of the electron spectrum inside the SNR following [Finke \& Dermer 2012]:

$$
\frac{\partial N(E, t)}{\partial t}+\frac{\partial}{\partial E} \dot{E} N(E, t)=Q(E, t)
$$

where 1) in the hadronic case, $\dot{E}$ is simply the adiabatic loss term $\dot{E}=k_{\mathrm{ad}} E / t$, with $k_{\mathrm{ad}}=1 ; 2$ ) for the electrons, besides adiabatic losses, we consider the loss terms due to synchrotron emission 
and inverse Compton scattering, modeled as in [Finke \& Dermer 2012]. We discretize the above equation on a two-dimensional grid, with linear spacing in time and log-spacing in energy. A second-order implicit scheme is implemented, in order to guarantee a stable solution regardless of the chosen timestep. In order to take into account the loss of particles upstream when the escape condition $l_{D}>\chi R_{\text {shock }}$ discussed above (with $\chi \approx 0.05 \ldots 0.1$ ) is fulfilled, we apply a posteriori to the solution an exponential cutoff at $E_{\max }$ (escape) for each timestep $i$. Concerning the electron evolution, the key parameter is the mean magnetic field inside the remnant $B_{\text {inside, }}$, which determines the synchrotron energy-loss rate. To estimate the mean field, we adopt this prescription: 1) During the early ejecta-dominated phase, we compute the internal energy by integrating the self-similar pressure profile taken from [Chevalier 1982] and assuming an equation of state $\varepsilon=3 / 2 P$ 2) After the transition to Sedov, we compute the internal energy - assuming the thin-shell approximation - as follows: $E_{\text {int }}=E_{\mathrm{SN}}-\frac{1}{2} M u_{S}^{2}$ with the same notation as eq.2.1. We set $E_{\mathrm{SN}}=10^{51} \mathrm{erg}$. $M$ is the total (swept + ejecta) mass. In general, we consider the interpolation between the two extreme regimes.

Given this recipe, we compute consistently the average magnetic field inside the remnant, and therefore the synchrotron energy-loss term for the electrons at all times. The final result of our computation is the complete time evolution of the electron and proton spectra.

\section{Results}

We discuss here our results on the time evolution of the gamma-ray emission from a type I and type II remnant. Once we have computed the evolution of the electron and proton spectra as described in the previous paragraph, we use the NAIMA public code ${ }^{2}$ to compute the corresponding non-thermal emission due to synchrotron, inverse Compton, and $\pi^{0}$ decay. Our main results are
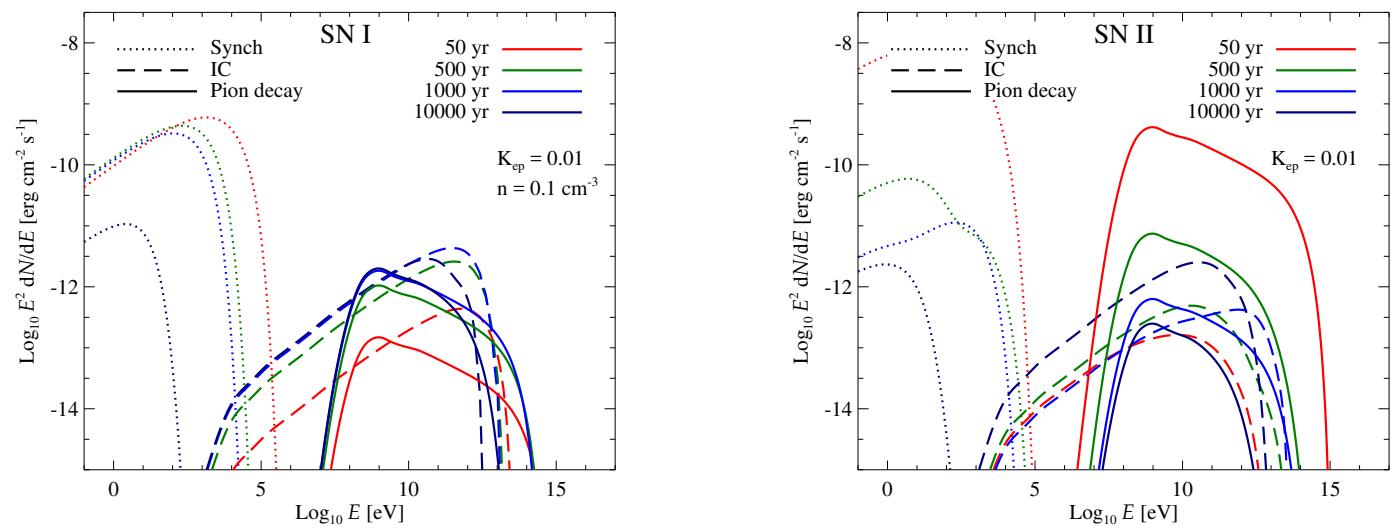

Figure 2: Typical evolution of the gamma-ray spectrum of a supernova remnant of type I and II for our reference values of the relevant parameters.

shown in Fig. 2 and 3. First of all, we consider two benchmark cases for the evolution of a type Ia and a type II SNR (see table 1 for a summary of the parameters adopted, and fig. 2 for the spectral

\footnotetext{
${ }^{2}$ https://github.com/zblz/naima
} 

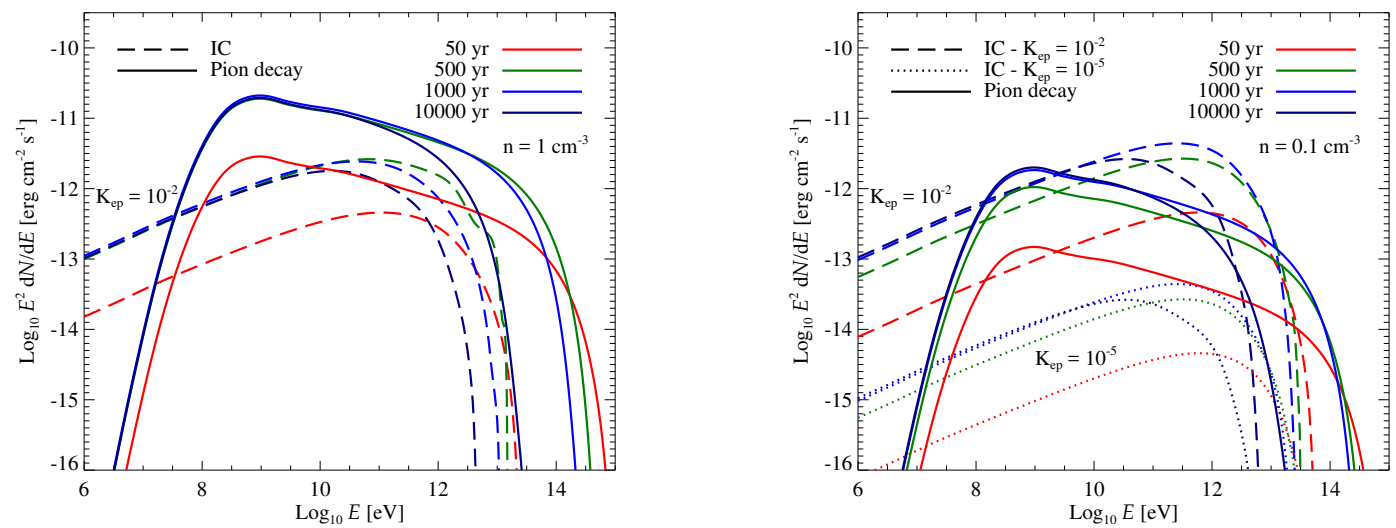

Figure 3: Impact of the gas density and $K_{e p}$ on the gamma-ray spectra of type I SNRs.

evolution: left and right panel correspond to type Ia and II). The gamma-ray emission from $\mathrm{MeV}$ to $\mathrm{PeV}$ is shown for some relevant reference ages: $50 \mathrm{y}$ (when the acceleration is expected to be efficient all the way up to $\mathrm{PeV}$ energies), $500 \mathrm{y}$ (comparable to the age of the well-known remnants Cas A and Tycho, $10^{3} \mathrm{y}$ (comparable with the age of RX J1713.7-3946, and $10^{4} \mathrm{y}$ (a late time when the remnant is at the end of the Sedov-Taylor phase). The different spectral shapes of the IC and $\pi^{0}$ emission are clearly seen in the plot. We remark that the relevance of the well-known characteristic feature below the $\pi^{0}$ mass scale range associated to hadronic emission (the so called pion bump) mainly depends on: 1) the ratio between the number density of electrons and protons $\left(K_{e p}\right)$; 2) the gas density of the surrounding medium. For the benchmark choice $\left(K_{e p}=0.01\right.$, $\left.n_{\text {gas }}=0.1 \mathrm{~cm}^{-3}\right)$, the pion bump can be noticed in the type I gamma-ray spectrum; for type II, it is clearly dominant at early times ( $<1$ century). The other key feature of interest in these spectra is the evolution of high-energy cutoff. Consistently to what we showed in the previous sections, the type II SNR - for the benchmark scenario and the default values of the parameters considered here - is a bright gamma-ray emitter all the way up to energies consistent with a CR population extending up to $\mathrm{PeV}$ (i.e., a PeVatron) during the early stage of its evolution, until $\sim 100 \mathrm{y}$. The IC spectrum shown in Fig. 2, in particular, shows a clear PeV cutoff at $t=50 \mathrm{y}$ (red solid line). On the other hand, the type I SNR is unable to reach such extreme energies, as far as the default setup and parametrization we are considering here is concerned. With these results at hand, we investigate now the impact of the most relevant parameters on both the pion bump and the presence of the Pevatron phase (and its duration). We see in fig. 3 how the pion bump is highly enhanced (and, vice $v e r s a$, heavily suppressed) for values of the gas density as large as $1 \mathrm{~cm}^{-3}$ and values of $K_{e p}$ as low as $10^{-5}$. As far the PeVatron issue is concerned, we remark that the large values of $E_{\max }$ reached by the type II SNRs in our computation are mainly due to: 1) The effectiveness of the magnetic field amplification, which is under debate especially for steep spectra, as recently summarized e.g. in [Gabici et al. 2016]. 2) The presence of a large gas density in the interstellar medium around the remnant. Since we are assuming that $3.5 \%$ of the shock ram pressure $P_{\mathrm{ram}}=\rho(r) u_{\mathrm{w}}(r)$ in converted into magnetic energy, and given that $\rho \propto \dot{M} / r^{2}$ in the supergiant wind, this condition is satisfied only at small radii, hence early times, for large enough values of $\dot{M}$. 


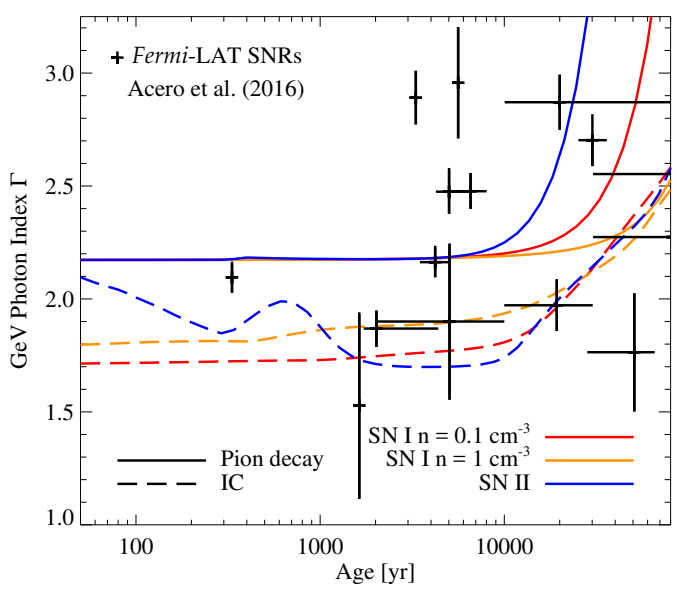

Figure 4: Predicted GeV spectral index in several scenarios, compared to current Fermi-LAT data.

Our framework offers the opportunity to perform systematic comparisons with collections of data on a diverse population of supernova remnants of different type and age. As an example, we show in Fig. 4 the predicted evolution of the GeV spectral index, in several benchmark cases, with the current Fermi-LAT data taken from [Acero et al. 2016] (interacting SNRs were removed from the sample). Although current data are not very constraining, future CTA observation in the TeV region of a wider sample of objects will allow to test the trends outlined in this work.

\section{Conclusions}

We have performed a systematic study about the time evolution of the electron and proton spectrum inside a SNR and the associated gamma-ray emission, with the future prospect of a detailed comparison with an extended and accurate set of data regarding a large population of young and middle-aged SNR. To this aim, we developed a numerical tool designed to compute the maximal energy of accelerated particles as a function of a well-defined set of free parameters, we computed the evolution of CR spectra accordingly, and finally provided a prediction for the radio and gammaray spectra at different times, and for the evolution of the GeV spectral index. We paid particular attention to the modeling of the magnetic field amplification: We followed a phenomenological approach driven by current X-ray measurements and discussed the problems with the theoretical scenarios proposed so far in this context. We outlined the dependence of the most relevant features, i.e. the presence and duration of the PeVatron phase and the significance of the hadronic pion bump, on the relevant parameters related to the SN event and the ambient medium. Finally, we briefly discussed the comparison with current Fermi-LAT data on the GeV spectral index.

\section{References}

[Baade \& Zwicky 1934] Baade, W.,, \& Zwicky, F. 1934, Contributions from the Mount Wilson

Observatory, vol. 3, pp.79-83, 3, 79 
[Ter Haar 1950] Ter Haar, D. 1950, Reviews of Modern Physics, 22, 119

[Morrison 1957] Morrison, P. 1957, Reviews of Modern Physics, 29, 235

[Blandford \& Ostriker 1978] Blandford, R. D., \& Ostriker, J. P. 1978, ApJL, 221, L29

[Bell 1978] Bell, A. R. 1978, MNRAS, 182, 147

[Axford et al. 1977] Axford, W. I., Leer, E., \& Skadron, G. 1977, International Cosmic Ray Conference, 11,132

[Krymskii 1977] Krymskii, G. F. 1977, Akademiia Nauk SSSR Doklady, 234, 1306

[HESS Collaboration et al. 2016] HESS Collaboration, Abramowski, A., Aharonian, F., et al. 2016, Nature, 531, 476

[Gaggero et al. 2017] Gaggero, D., Grasso, D., Marinelli, A., Taoso, M., \& Urbano, A. 2017, arXiv: 1702.01124

[Jouvin et al. 2017] Jouvin, L., Lemière, A., \& Terrier, R. 2017, MNRAS, 467, 4622

[Chevalier 1982] Chevalier, R. A. 1982, ApJ, 258, 790

[Bell 2004] Bell, A.R., 2004, MNRAS, 353, 550

[Bistnovatyi-Kogan 1995] Bisnovatyi-Kogan, G.S., Silich, S.A., 1995, Rev. Mod. Phys., 67, 661

[Castor et al. 1975] Castor, J., McCray, R., Weaver, R., 1975, ApJ Lett., 200, L107

[Chevalier 1982] Chevalier, R.A., 1982, ApJ, 258, 790

[Cristofari et al. 2013] Cristofari, P., Gabici, S., Casanova, S., Terrier, R., Parizot, E., 2013, MNRAS, 434, 2748

[Drury 1983] Drury, L.O’C., 1983, Phys. Rep., 46, 973

[Drury \& Downes 2012] Drury, L.O’C., Downes, T.P. 2012, MNRAS, 427, 2308

[Giacalone 2007] Giacalone, J., \& Jokipii, J. R. 2007, ApJL, 663, L41

[Finke \& Dermer 2012] Finke, J.D., Dermer, C.D., 2012, ApJ, 751, 65

[Hillas 2005] Hillas, A.M., 2005, J. Phys. G: Nucl. Part. Phys., 31, R95

[Lagage \& Cesarsky 1983] Lagage, P.O., Cearsky, C.., 1983, A\&A, 125, 249

[Ostriker \& McKee 1988] Ostriker, J.P., McKee, C.F., 1988, Rev. Mod. Phys., 60, 1

[Parizot et al. 2004] Parizot, E., Marcowith, A., van der Swaluw, E., Bykov, A.M., Tatischeff, V., 2004, A\&A, 424, 747

[Ptuskin \& Zirakashvili 2005] Ptuskin, V.S., Zirakashvili, V.N., 2005, A\&A, 429, 755

[Schure \& Bell 2013] Schure, K.M., Bell, A.R., 2013, MNRAS, 435, 1174

[Vannoni et al. 2009] Vannoni, G., Gabici, S., Aharonian, F.A., 2009, A\&A, 497, 17

[Vink 2012] Vink, J., 2012, A\&A Rev., 20, 49

[Völk et al. 2005] Völk, H.J., Berezhko, E.G., Ksenofontov, L.T., 2005, A\&A, 433, 229

[Zirakashvili \& Ptuskin 2012] Zirakashvili, V.N., Ptuskin, V.S., 2012, Astropart. Phys., 39, 12

[Finke \& Dermer 2012] Finke J.D. and Dermer C.D., 2012, Astrophys. J. 751, 65

[Gabici et al. 2016] Gabici S., Gaggero D., Zandanel F., 2016, arXiv:1610.07638

[Acero et al. 2016] Acero, F., Ackermann, M., Ajello, M., et al. 2016, ApJS, 224, 8 